PROCEEDINGS OF THE

AMERICAN MATHEMATICAL SOCIETY

Volume 138, Number 11, November 2010, Pages 3965-3974

S 0002-9939(2010)10396-9

Article electronically published on May 14, 2010

\title{
CLASSIFYING CANTOR SETS BY THEIR FRACTAL DIMENSIONS
}

\author{
CARLOS A. CABRELLI, KATHRYN E. HARE, AND URSULA M. MOLTER
}

(Communicated by Michael T. Lacey)

\begin{abstract}
In this article we study Cantor sets defined by monotone sequences, in the sense of Besicovich and Taylor. We classify these Cantor sets in terms of their $h$-Hausdorff and $h$-packing measures, for the family of dimension functions $h$, and characterize this classification in terms of the underlying sequences.
\end{abstract}

\section{INTRODUCTION}

A natural way to classify compact subsets $E \subseteq \mathbb{R}$ of Lebesgue measure zero is by their Hausdorff or packing dimension. This is a crude measurement, however, which often does not distinguish salient features of the set. For a finer classification, one could consider the family of $h$-Hausdorff measures, $H^{h}$, and $h$-packing measures, $P^{h}$, where $h$ belongs to the set of dimension functions $\mathcal{D}$, defined in Section 2.

Definition 1.1. By the dimension partition of a set $E$, we mean a partition of $\mathcal{D}$ into (six) sets $\mathcal{H}_{\alpha}^{E} \cap \mathcal{P}_{\beta}^{E}$, for $\alpha \leq \beta \in\{0,1, \infty\}$, where

$$
\begin{aligned}
& \mathcal{H}_{\alpha}^{E}=\left\{h \in \mathcal{D}: H^{h}(E)=\alpha\right\} \text { for } \alpha=0, \infty, \\
& \mathcal{H}_{1}^{E}=\left\{h \in \mathcal{D}: 0<H^{h}(E)<\infty\right\},
\end{aligned}
$$

and $\mathcal{P}_{\beta}^{E}$ is defined similarly, but with $h$-packing measure replacing $h$-Hausdorff measure.

Sets which have the same dimension partition will have the same Hausdorff and packing dimensions; however the converse is not necessarily true.

We call a compact, perfect, totally disconnected, measure zero subset of the real line a Cantor set. There is a natural way (see Section 3.1) to associate to each summable, non-increasing sequence $a=\left\{a_{n}\right\} \subseteq \mathbb{R}^{+}$a unique Cantor set $C_{a}$ having gaps with lengths corresponding to the terms $a_{n}$. The study of the dimension of Cantor sets by means of its gaps was initiated by Besicovich and Taylor [1. In fact, the Hausdorff and packing dimensions of $C_{a}$ can be calculated in terms of the tails of the sequence $a$, the numbers $r_{n}^{(a)}=\sum_{i \geq n} a_{i}$, for $n \in \mathbb{N}$. In this paper we show

Received by the editors May 11, 2009 and, in revised form, January 15, 2010.

2010 Mathematics Subject Classification. Primary 28A78, 28A80.

Key words and phrases. Hausdorff dimension, packing dimension, Cantor set, cut-out set.

The first and third authors were partially supported by Grants UBACyT X149 and X028 (UBA), PICT 2006-00177 (ANPCyT), and PIP 112-200801-00398 (CONICET).

The second author was partially supported by NSERC. 
that the classification of the sets $C_{a}$ according to their dimension partitions can be characterized in terms of properties of their tails.

We introduce a partial ordering, $\preceq$, on the set of dimension functions, which preserves the order of Hausdorff and packing $h$-measures. A set $E$ is said to be $h$-regular if $h \in \mathcal{H}_{1}^{E} \cap \mathcal{P}_{1}^{E}$. If this holds for $h=x^{s}$, then $E$ is said to be $s$-regular, and in that case the Hausdorff and packing dimensions of $E$ are both $s$. Although not every Cantor set $C_{a}$ of dimension $s$ is an $s$-regular set, we show that one can always find a dimension function $h$ such that $C_{a}$ is an $h$-regular set. This completes arguments begun in [2] and [5]. The dimension functions for which a given Cantor set, $C_{a}$, is regular form an equivalence class under this ordering and are called the dimension functions associated to the sequence $a$.

We prove that two Cantor sets $C_{a}, C_{b}$ have the same dimension partition if and only if their associated dimension functions, $h_{a}, h_{b}$, are equivalent. More generally, we prove that $C_{a}$ and $C_{b}$ have the same dimension partition if and only $a$ and $b$ are weak tail-equivalent (see Definition 4.2).

Furthermore, we show that the weak tail-equivalence can be replaced by the stronger tail-equivalence relation only when the associated dimension functions have inverse with the doubling property.

\section{Dimension FunCtions And MeAsures}

A function $h$ is said to be increasing if $h(x)<h(y)$ for $x<y$ and doubling if there exists $\tau>0$ such that $h(x) \geq \tau h(2 x)$ for all $x$ in the domain of $h$. We will say that a function $h:(0, A] \rightarrow(0, \infty]$ is a dimension function if it is continuous, increasing, doubling and $h(x) \rightarrow 0$ as $x \rightarrow 0$. We denote by $\mathcal{D}$ the set of dimension functions. A typical example of a dimension function is $h_{s}(x)=x^{s}$ for some $s>0$.

Given any dimension function $h$, one can define the $h$-Hausdorff measure of a set $E, H^{h}(E)$ in the same manner as the Hausdorff $s$-measure (see [6]): Let $|B|$ denote the diameter of a set $B$. Then

$$
H^{h}(E)=\lim _{\delta \rightarrow 0^{+}}\left(\inf \left\{\sum h\left(\left|E_{i}\right|\right): \bigcup E_{i} \subseteq E,\left|E_{i}\right| \leq \delta\right\}\right) .
$$

The Hausdorff $s$-measure is the special case when $h=h_{s}$. In terms of our notation, the Hausdorff dimension of $E$ is given by

$$
\operatorname{dim}_{H} E=\sup \left\{s: h_{s} \in \mathcal{H}_{\infty}^{E}\right\}=\inf \left\{s: h_{s} \in \mathcal{H}_{0}^{E}\right\} .
$$

The $h$-packing pre-measure is defined as in [7. First, recall that a $\delta$-packing of a given set $E$ is a disjoint family of open balls centered at points of $E$ with diameters less than $\delta$. The h-packing pre-measure of $E$ is defined by

$$
P_{0}^{h}(E)=\lim _{\delta \rightarrow 0^{+}}\left(\sup \left\{\sum h\left(\left|B_{i}\right|\right):\left\{B_{i}\right\}_{i} \text { is a } \delta \text {-packing of } E\right\}\right) .
$$

It is clear from the definition that the set function $P_{0}^{h}$ is monotone, but it is not a measure because it is not $\sigma$-sub-additive. The $h$-packing measure is obtained by a standard argument:

$$
P^{h}(E)=\inf \left\{\sum_{i=1}^{\infty} P_{0}^{h}\left(E_{i}\right): E=\bigcup E_{i}\right\} .
$$

The pre-packing dimension is the critical index given by the formula

$$
\operatorname{dim}_{P_{0}} E=\sup \left\{s: P_{0}^{h_{s}}(E)=\infty\right\}=\inf \left\{s: P_{0}^{h_{s}}(E)=0\right\}
$$


and is known to coincide with the upper box dimension [7. Clearly, $P^{h}(E) \leq$ $P_{0}^{h}(E)$, and as $h$ is a doubling function, $H^{h}(E) \leq P^{h}(E)$ (see [8]). The packing dimension is defined analogously and, like the Hausdorff dimension, can be obtained as

$$
\operatorname{dim}_{P}(E)=\sup \left\{s: h_{s} \in \mathcal{P}_{\infty}^{E}\right\}=\inf \left\{s: h_{s} \in \mathcal{P}_{0}^{E}\right\} .
$$

Finally, we note that the set $E$ is said to be $h$-regular if $0<H^{h}(E) \leq P^{h}(E)<\infty$ and $s$-regular if it is $h_{s}$-regular. The set $\mathcal{H}_{1}^{E} \cap \mathcal{P}_{1}^{E}$ consists of the $h$-regular functions of $E$.

We are interested in comparing different dimension functions.

Definition 2.1. Suppose $f, h: X \rightarrow(0, A]$. We will say $f \preceq h$ if there exists a positive constant $c$ such that

$$
f(x) \leq \operatorname{ch}(x) \text { for all } x .
$$

We will say $f$ is equivalent to $h$, and write $f \equiv h$, if $f \preceq h$ and $h \preceq f$.

This defines a partial ordering that is consistent with the usual pointwise ordering of functions. It is not quite the same as the ordering defined in [5], but we find it to be more natural.

Note that the definition of equivalence of functions, when applied to sequences, implies that $x=\left\{x_{n}\right\}$ and $y=\left\{y_{n}\right\}$ are equivalent if and only if there exist $c_{1}, c_{2}>0$ such that $c_{1} \leq x_{n} / y_{n} \leq c_{2}$ for all $n$.

The following easy result is very useful and also motivates the definition of $\preceq$.

Proposition 2.2. Suppose $h_{1}, h_{2} \in \mathcal{D}$ and $h_{1} \preceq h_{2}$. There is a positive constant $c$ such that for any Borel set $E$,

$$
H^{h_{1}}(E) \leq c H^{h_{2}}(E) \text { and } P_{(0)}^{h_{1}}(E) \leq c P_{(0)}^{h_{2}}(E) .
$$

Proof. Suppose $h_{1}(x) \leq \operatorname{ch}_{2}(x)$ for all $x$. For any $\delta>0$,

$$
\begin{aligned}
H_{\delta}^{h_{1}}(E) & =\inf \left\{\sum h_{1}\left(\left|U_{i}\right|\right), E \subseteq \bigcup_{i} U_{i},\left|U_{i}\right|<\delta\right\} \\
& \leq \operatorname{cinf}\left\{\sum h_{2}\left(\left|U_{i}\right|\right), E \subseteq \bigcup_{i} U_{i},\left|U_{i}\right|<\delta\right\}=c H_{\delta}^{h_{2}}(E) .
\end{aligned}
$$

The arguments are similar for packing pre-measure.

Corollary 2.3. If $h_{1}, h_{2} \in \mathcal{D}$ and $h_{1} \equiv h_{2}$, then for any Borel set $E, h_{1}$ and $h_{2}$ belong to the same set $\mathcal{H}_{\alpha}^{E} \cap \mathcal{P}_{\beta}^{E}$.

\section{Cantor sets associated to sequences}

3.1. Cantor sets $C_{a}$. Each Cantor set is completely determined by its gaps, the bounded convex components of the complement of the set. To each summable sequence of positive numbers, $a=\left\{a_{n}\right\}_{n=1}^{\infty}$, we can associate a unique Cantor set with gaps whose lengths correspond to the terms of this sequence.

To begin, let $I$ be an interval of length $\sum_{n=1}^{\infty} a_{n}$. We remove from $I$ an interval of length $a_{1}$. Then we remove from the left remaining interval an interval of length $a_{2}$ and from the right an interval of length $a_{3}$. Iterating this procedure, it is easy to see that we are left with a Cantor set which we will call $C_{a}$.

Observe that as $\sum a_{k}=|I|$, there is only one choice for the location of each interval to be removed in the construction. More precisely, the position of the first 
gap we place (of length $a_{1}$ ) is uniquely determined by the property that the length of the remaining interval on its left should be $a_{2}+a_{4}+a_{5}+a_{8}+\ldots$ Therefore, this construction defines the Cantor set unequivocally. As an example, if we take $a_{n}=1 / 3^{k}$ for $n=2^{k-1}, \ldots, 2^{k}-1, k=1,2, \ldots$, the classical middle-third Cantor set is produced. In this case the sequence $\left\{a_{n}\right\}$ is non-increasing.

This is also the case for any central Cantor set with fixed rate of dissection, those Cantor sets constructed in a similar manner as the classical 1/3 Cantor set but replacing $1 / 3$ by a number $0<a<1 / 2$, where $a$ is the ratio of the length of an interval of one step and the length of its parent interval.

We should remark that the order of the sequence is important. Different rearrangements could correspond to different Cantor sets, even of different dimensions; however, if two sequences correspond to the same Cantor set, one is clearly a rearrangement of the other. From here on we will assume that our sequence is positive, non-increasing and summable.

Given such a sequence, $a=\left\{a_{n}\right\}$, we denote by $r_{n}=r_{n}^{(a)}$ the tail of the series:

$$
r_{n}=\sum_{j \geq n} a_{j}
$$

The Hausdorff and pre-packing dimensions of $C_{a}$ are given by the formulas (see [2] and [5])

$$
\operatorname{dim}_{H} C_{a}=\varliminf_{n \rightarrow \infty} \frac{-\log n}{\log \left(r_{n}^{(a)} / n\right)} \text { and } \operatorname{dim}_{P_{0}} C_{a}=\varlimsup_{n \rightarrow \infty} \frac{-\log n}{\log \left(r_{n}^{(a)} / n\right)} .
$$

Motivated by the analogous result in [1] for $s$-Hausdorff measure, it was shown in [5] that for any dimension function $h$, the Hausdorff $h$-measure and $h$-packing premeasure of the Cantor set $C_{a}$ are determined by the limiting behaviour of $h\left(r_{n} / n\right)$.

Theorem 3.1 ([5, Prop. 4.1, Thm. 4.2]). For any $h \in \mathcal{D}$,

$$
\frac{1}{4} \underline{\lim }_{n \rightarrow \infty} n h\left(\frac{r_{n}^{(a)}}{n}\right) \leq H^{h}\left(C_{a}\right) \leq 4 \underline{\lim _{n \rightarrow \infty}} n h\left(\frac{r_{n}^{(a)}}{n}\right),
$$

and similarly for $P_{0}^{h}\left(C_{a}\right)$, but with lim sup replacing lim inf.

This suggests that it will be of interest to study the following class of functions:

Definition 3.2. We will say that an increasing, continuous function $h:(0, A] \rightarrow$ $(0, \infty]$ is associated to the sequence $a$ (or to the Cantor set $C_{a}$ ) if the sequence $\left\{h\left(r_{n}^{(a)} / n\right)\right\}$ is equivalent to the sequence $\{1 / n\}$.

One can check that any function $h_{a}$ associated to the sequence $a$ is a doubling function and thus belongs to $\mathcal{D}$. Indeed, if

$$
c_{1} h_{a}\left(\frac{r_{n}}{n}\right) \leq \frac{1}{n} \leq c_{2} h_{a}\left(\frac{r_{n}}{n}\right) \text { for all } n
$$

and $r_{n} / n \leq x \leq r_{(n-1)} /(n-1)$, then by monotonicity,

$$
h_{a}(2 x) \leq h_{a}\left(\frac{2 r_{n-1}}{n-1}\right) \leq h_{a}\left(\frac{r_{[(n-1) / 2]}}{(n-1) / 2}\right) \leq \frac{4}{c_{1} n} \leq \frac{4 c_{2}}{c_{1}} h_{a}(x) .
$$

In the special case that $a=\left\{n^{-1 / s}\right\}$ for some $0<s<1$, it is known (see [3]) that $C_{a}$ has Hausdorff dimension $s$ and $0<H^{h_{s}}\left(C_{a}\right)<\infty$. One can easily see that any function associated to $C_{a}$ is equivalent to $x^{s}$. This generalizes to arbitrary 
associated (dimension) functions, so we can speak of 'the' associated dimension function.

Lemma 3.3. If $h$ is associated to the sequence $a$ and $g \in \mathcal{D}$, then $h \equiv g$ if and only if $g$ is also associated to $a$.

Proof. Suppose $g$ is associated to $a$. Let $b_{n}=r_{n}^{(a)} / n$ and $b_{n+1} \leq x \leq b_{n}$. As $\left\{h\left(b_{n}\right)\right\} \equiv\left\{g\left(b_{n}\right)\right\} \equiv\{1 / n\}$ and $g$ is monotonic,

$$
c_{1} h\left(b_{n+1}\right) \leq g\left(b_{n+1}\right) \leq g(x) \leq g\left(b_{n}\right) \leq c_{2} h\left(b_{n}\right)
$$

for all $n$ and for suitable constants $c_{1}, c_{2}$. Thus

$$
c_{1}^{\prime} \leq c_{1} \frac{h\left(b_{n+1}\right)}{h\left(b_{n}\right)} \leq \frac{g(x)}{h(x)} \leq c_{2} \frac{h\left(b_{n}\right)}{h\left(b_{n+1}\right)} \leq c_{2}^{\prime} .
$$

The other implication is straightforward.

3.2. Packing dimension of Cantor sets $C_{a}$. The pre-packing dimension and packing pre-measure always majorizes the packing dimension and packing measure, and the strict inequality can hold. For example, it is an easy exercise to see that the packing dimension of the countable set $\{1 / n\}_{n=1}^{\infty}$ is 0 , but the pre-packing dimension equals $1 / 2$. This phenomena does not happen for the Cantor sets $C_{a}$. To prove this, we begin with a technical result which generalizes [4, Prop. 2.2].

Lemma 3.4. Let $\mu$ be a finite, regular, Borel measure and let $h \in \mathcal{D}$. If

$$
\varliminf_{r \rightarrow 0} \frac{\mu\left(B\left(x_{0}, r\right)\right)}{h(r)}<c \text { for all } x_{0} \in E,
$$

then

$$
P^{h}(E) \geq \frac{\mu(E)}{c}
$$

Proof. We need to prove that for any partition $\bigcup_{i=1}^{\infty} E_{i}=E$, we have $\sum_{i=1}^{\infty} P_{0}^{h}\left(E_{i}\right)$ $\geq \mu(E) / c$. Since $\mu(E) \leq \sum \mu\left(E_{i}\right)$, it is enough to prove that $P_{0}^{h}\left(E_{i}\right) \geq \mu\left(E_{i}\right) / c$ for each $i$.

Without loss of generality assume $E_{i}=E$, and we will show that for each $\delta>0$,

$$
P_{0, \delta}^{h}(E) \equiv \sup \left\{\sum h\left(\left|B_{i}\right|\right):\left\{B_{i}\right\}_{i} \text { is a } \delta \text {-packing of } E\right\} \leq \frac{\mu(E)}{c} .
$$

Consider the collection of balls $B(x, r)$, with $x \in E$ and $\mu(B(x, r))<\operatorname{ch}(r)$, where $r \leq \delta$. The hypothesis ensures that for each $x \in E$ there are balls $B(x, r)$ in the collection, with $r$ arbitrarily small. By the Vitali covering lemma, there are disjoint balls from this collection, $\left\{B_{i}\right\}_{i=1}^{\infty}$, with $\mu\left(E \backslash \cup B_{i}\right)=0$. Thus

$$
P_{0, \delta}^{h}(E) \geq \sum h\left(\left|B_{i}\right|\right) \geq \frac{1}{c} \sum \mu\left(B_{i}\right)=\frac{1}{c} \mu\left(\bigcup B_{i}\right)=\frac{1}{c} \mu(E) .
$$

We now specialize to the case of Cantor sets $C_{a}$ associated to a non-increasing, summable sequence $a=\left\{a_{j}\right\}$. If we use the notation $\left\{I_{j}^{(k)}\right\}_{1 \leq j \leq 2^{k}}$ for the (remaining) intervals at step $k$ in the Cantor set construction, then the sequence of lengths of these intervals, $\left\{\left|I_{j}^{(k)}\right|\right\}_{(k, j)}$, with $1 \leq j \leq 2^{k}, k \geq 1$ is (lexicographically) non-increasing. Hence the length of any Cantor interval of step $k$ is at least the length of any Cantor interval of step $k+1$. This observation, together with the lemma above, is the key idea needed to prove that infinite (or positive) pre-packing measure implies infinite (respectively, positive) packing measure for the sets $C_{a}$. Of course, the other implication holds for all sets. 
Theorem 3.5. Suppose $a=\left\{a_{j}\right\}$ is a summable, non-increasing sequence with associated Cantor set $C_{a}$ and $h \in \mathcal{D}$. If $P_{0}^{h}\left(C_{a}\right)=\infty$, then $P^{h}\left(C_{a}\right)=\infty$, while if $P_{0}^{h}\left(C_{a}\right)>0$, then $P^{h}\left(C_{a}\right)>0$.

Proof. Let $b_{n}=r_{n}^{(a)} / n$. By Theorem 3.1, $P_{0}^{h}\left(C_{a}\right)=\infty$ implies $\left\lceil\lim n h\left(b_{n}\right)=\infty\right.$ and $P_{0}^{h}\left(C_{a}\right)>0$ implies $\overline{\lim } n h\left(b_{n}\right)>0$. Since $h$ is increasing, if $2^{k} \leq n \leq 2^{k+1}$, then

$$
n h\left(b_{n}\right) \leq n h\left(b_{2^{k}}\right) \leq 2^{k+1} h\left(b_{2^{k}}\right) .
$$

Therefore, $\varlimsup_{k \rightarrow \infty} 2^{k} h\left(b_{2^{k}}\right)=\infty$ in the first case and is (strictly) positive in the second.

The tail term, $r_{2^{k}}^{(a)}$, is the sum of gaps created at level $k+1$ or later, which in turn is equal to the sum of the lengths of the step $k$ intervals. Thus $r_{2^{k}} / 2^{k}$, the average length of a step $k$ interval, is at most the length of the shortest interval of step $k-1$ and at least the length of longest interval of step $k+1$. As $h$ is increasing,

$$
h\left(\left|I_{1}^{(k)}\right|\right) \geq h\left(b_{2^{k}}\right) \geq h\left(\left|I_{1}^{(k+1)}\right|\right) .
$$

Let $\mu$ be the (uniform) Cantor measure on $C_{a}$ (constructed as a limiting process, assigning at each step $k$ the measure $\mu_{k}$ such that $\mu_{k}\left(I_{j}^{k}\right)=2^{-k}$ and then taking the weak*-limit).

Fix $x_{0} \in C_{a}$ and $r>0$. Suppose $k$ is the minimal integer such that $B\left(x_{0}, r\right)$ contains a step $k$ interval. The minimality of $k$ ensures that $B\left(x_{0}, r\right)$ can intersect at most 5 step $k$ intervals. Thus $\mu\left(B\left(x_{0}, r\right)\right) \leq 52^{-k}$. Also, if $I_{j}^{(k)}$ is a step $k$ interval contained in $B\left(x_{0}, r\right)$, then $2 r \geq\left|I_{j}^{(k)}\right|$. Since $h$ is a doubling function,

$$
h(r) \geq \tau h(2 r) \geq \tau h\left(\left|I_{j}^{(k)}\right|\right) \geq \tau h\left(b_{2^{k+1}}\right)
$$

for some $\tau>0$.

Combining these facts, we see that

$$
\frac{\mu\left(B\left(x_{0}, r\right)\right)}{h(r)} \leq \frac{5 \cdot 2^{-k}}{\tau h\left(b_{2^{k+1}}\right)}=\frac{10}{\tau 2^{k+1} h\left(b_{2^{k+1}}\right)} .
$$

Thus if $P_{0}^{h}\left(C_{a}\right)=\infty$, then

$$
\varliminf_{r \rightarrow 0} \frac{\mu\left(B\left(x_{0}, r\right)\right)}{h(r)}=0,
$$

while if $P_{0}^{h}\left(C_{a}\right)>0$, then

$$
c_{0}=\lim _{r \rightarrow 0} \frac{\mu\left(B\left(x_{0}, r\right)\right)}{h(r)}<\infty .
$$

Applying Lemma 3.4 we conclude that in the first case, $P^{h}\left(C_{a}\right) \geq \mu\left(C_{a}\right) / c$ for every $c>0$ and therefore $P^{h}\left(C_{a}\right)=\infty$, while $P^{h}\left(C_{a}\right) \geq \mu\left(C_{a}\right) / c_{0}>0$ in the second case.

Corollary 3.6. (i) For any dimension function $h, P_{0}^{h}\left(C_{a}\right)=0$ (or $\infty$ ) if and only if $P^{h}\left(C_{a}\right)=0$ (resp. $\infty$ ) and $0<P_{0}^{h}\left(C_{a}\right)<\infty$ if and only if $0<P^{h}\left(C_{a}\right)<\infty$.

(ii) The packing and pre-packing dimensions of the Cantor set $C_{a}$ coincide.

Theorem 3.1 was used in [5] to give sufficient conditions for two dimension functions to be equivalent. Together with Theorem 3.5, we can obtain sufficient conditions for comparability. 
Proposition 3.7. Suppose $f, h \in \mathcal{D}$. If $H^{h}\left(C_{a}\right)>0$ and $P^{f}\left(C_{a}\right)<\infty$, then $f \preceq h$.

Proof. Let $b_{n}=r_{n}^{(a)} / n$. Since $H^{h}\left(C_{a}\right)>0$, Theorem 3.1 implies that there exists a constant $c_{1}>0$ such that for all sufficiently large $n, h\left(b_{n}\right) \geq c_{1} / n$. Similarly, the assumption that $P^{f}\left(C_{a}\right)<\infty$ implies $P_{0}^{f}\left(C_{a}\right)<\infty$ by our previous theorem, and therefore there is a constant $c_{2}<\infty$ with $f\left(b_{n}\right) \leq c_{2} / n$.

Now suppose $b_{n} \leq x<b_{n-1}$. By monotonicity, $f(x) \leq f\left(b_{n-1}\right)$ and $h(x) \geq$ $h\left(b_{n}\right)$. Hence

and therefore $f \preceq h$.

$$
\frac{f(x)}{h(x)} \leq \frac{c_{2} n}{c_{1}(n-1)} \leq 2 \frac{c_{2}}{c_{1}}<\infty
$$

The next result was obtained in [5. Thm. 4.4] with packing pre-measure replacing packing measure.

Corollary 3.8. Suppose $f, h \in \mathcal{D}$. If $0<H^{g}\left(C_{a}\right) \leq P^{g}\left(C_{a}\right)<\infty$ for $g=f$ and $h$, then $f \equiv h$.

\section{Classification of Cantor sets}

An immediate consequence of Theorems 3.1 and 3.5 is the following elegant description of the dimension partition for Cantor sets $C_{a}$.

Theorem 4.1. Suppose $a=\left\{a_{n}\right\}$ is a non-increasing, summable sequence of positive real numbers. Then

$$
\begin{aligned}
& \mathcal{H}_{\alpha}^{C_{a}}=\left\{h \in \mathcal{D}: \varliminf_{n \rightarrow \infty} n h\left(\frac{r_{n}^{(a)}}{n}\right)=\alpha\right\} \text { for } \alpha=0, \infty, \\
& \mathcal{H}_{1}^{C_{a}}=\left\{h \in \mathcal{D}: 0<\varliminf_{n \rightarrow \infty} n h\left(\frac{r_{n}^{(a)}}{n}\right)<\infty\right\},
\end{aligned}
$$

and similarly for $\mathcal{P}_{\beta}^{C_{a}}, \beta=0,1, \infty$, but with lim sup replaced by lim inf. (See Table 1.)

In particular, note that $C_{a}$ is $h$-regular if and only if $h$ is a dimension function associated to $a$.

TABle 1. Classification of functions in $\mathcal{D}$ for $C_{a}$

\begin{tabular}{c|c|c|c|}
\multicolumn{1}{c}{$P_{0}$} & \multicolumn{1}{c|}{$P_{1}$} \\
\cline { 2 - 3 }$H_{0}$ & $\begin{array}{l}\text { 0- } h \text { Hausdorff measure } \\
\text { 0- } h \text { Packing measure }\end{array}$ & $\begin{array}{l}0-h \text { Hausdorff measure } \\
h \text {-Packing set }\end{array}$ & $\begin{array}{l}0-h \text { Hausdorff measure } \\
\infty-h \text { Packing measure }\end{array}$ \\
\cline { 2 - 3 }$H_{1}$ & $h$-regular set & $\begin{array}{l}h \text {-Hausdorff set } \\
\infty-h \text { Packing measure }\end{array}$ \\
\cline { 2 - 3 }$H_{\infty}$ & & $\begin{array}{l}\infty-h \text { Hausdorff measure } \\
\infty-h \text { Packing measure }\end{array}$ \\
\hline
\end{tabular}


For the computation of dimensions the relevant behaviour of a sequence is that of its tail; thus we introduce the following definitions.

Definition 4.2. (a) We say two sequences $a, b$ are tail-equivalent if the sequences of tails $\left\{r_{n}^{(a)}\right\},\left\{r_{n}^{(b)}\right\}$ are equivalent.

(b) We say $a$ and $b$ are weak tail-equivalent if there are positive integers $j, k$ such that $r_{n}^{(a)} \geq r_{j n}^{(b)} / j$ and $r_{n}^{(b)} \geq r_{k n}^{(a)} / k$ for all $n$.

Obviously, if $a$ is tail-equivalent to $b$, then the dimension partitions of $C_{a}$ and $C_{b}$ are the same. Furthermore, equivalence of sequences implies tail-equivalence which implies weak tail-equivalence; however neither implication is reversible, as the next example illustrates.

Example 4.3. (a) Let $k_{1}=1$ and inductively define $n_{j}=2^{k_{j}+1}-2$ and $k_{j+1}=$ $n_{j}+k_{j}+1$. Set $a_{k_{j}}=2^{-k_{j}}, a_{n}=2^{-\left(2 k_{j}+1\right)}$ for $n=k_{j}+1, \ldots, n_{j}+k_{j}$, and set $b_{n}=2^{-2 k_{j}}$ for $n=k_{j}+1, \ldots, n_{j}+k_{j}$. Since $a_{k_{j}} / b_{k_{j}}=2^{k_{j}},\left\{a_{n}\right\}$ is not equivalent to $\left\{b_{n}\right\}$. However one can easily check that $\left\{a_{n}\right\}$ is tail equivalent to $\left\{b_{n}\right\}$.

(b) The sequence $\left\{e^{-n}\right\}$ is weak tail-equivalent to $\left\{e^{-2 n}\right\}$, but not tail-equivalent.

We are now ready to state and prove our classification result. For notational ease we write $\mathcal{H}_{\alpha}^{a}, \mathcal{P}_{\beta}^{a}$ rather than $\mathcal{H}_{\alpha}^{C_{a}}, \mathcal{P}_{\beta}^{C_{a}}$.

Theorem 4.4. Suppose $C_{a}$ and $C_{b}$ are Cantor sets associated to non-increasing, summable sequences $a, b$ of positive numbers. The following are equivalent:

(1) The dimension function associated to a is equivalent to the dimension function associated to $b$.

(2) $C_{a}$ and $C_{b}$ are $h$-regular for precisely the same set of dimension functions $h$.

(3) The dimension partitions associated with $C_{a}$ and $C_{b}$ coincide; i.e., $\mathcal{H}_{\alpha}^{a} \cap$ $\mathcal{P}_{\beta}^{a}=\mathcal{H}_{\alpha}^{b} \cap \mathcal{P}_{\beta}^{b}$ for all $\alpha \leq \beta \in\{0,1, \infty\}$.

(4) The sequence $a$ is weak tail-equivalent to the sequence $b$.

Proof. $(2 \Rightarrow 1)$ Since $C_{b}$ is $h_{b}$-regular, $C_{a}$ must also be $h_{b}$-regular. Thus $h_{a}, h_{b} \in$ $\mathcal{H}_{1}^{a} \cap \mathcal{P}_{1}^{a}$. But all functions in $\mathcal{H}_{1}^{a} \cap \mathcal{P}_{1}^{a}$ are equivalent by Corollary 3.8

$(3 \Rightarrow 2)$ is obvious since $(2)$ could be stated as $\mathcal{H}_{1}^{a} \cap \mathcal{P}_{1}^{a}=\mathcal{H}_{1}^{b} \cap \mathcal{P}_{1}^{b}$.

$(4 \Rightarrow 3)$ It is easy to see that if there exists an integer $j$ such that $r_{n}^{(a)} \geq r_{j n}^{(b)} / j$, then $\mathcal{H}_{0}^{a} \subseteq \mathcal{H}_{0}^{b}$ and $\mathcal{H}_{\infty}^{b} \subseteq \mathcal{H}_{\infty}^{a}$.

To see that $\mathcal{P}_{\infty}^{b} \subseteq \mathcal{P}_{\infty}^{a}$, suppose there is a sequence $\left\{n_{i}\right\}$ with $\lim _{i} n_{i} h\left(r_{n_{i}}^{(b)} / n_{i}\right)=$ $\infty$. Let $m_{i}=\left[\frac{n_{i}}{j}\right]$, where $[z]$ means the integer part of $z$. As $n_{i} \geq j m_{i}$ and $r_{n} / n$ is decreasing,

Since $h$ is increasing

$$
\frac{r_{n_{i}}^{(b)}}{n_{i}} \leq \frac{r_{j m_{i}}^{(b)}}{j m_{i}} \leq \frac{r_{m_{i}}^{(a)}}{m_{i}}
$$

$$
2 j m_{i} h\left(\frac{r_{m_{i}}^{(a)}}{m_{i}}\right) \geq n_{i} h\left(\frac{r_{n_{i}}^{(b)}}{n_{i}}\right) \rightarrow \infty
$$

therefore, $\varlimsup \lim n h\left(\frac{r_{n}^{(a)}}{n}\right)=\infty$. A similar argument proves $\mathcal{P}_{0}^{a} \subseteq \mathcal{P}_{0}^{b}$.

Consequently, if $a$ is weak tail-equivalent to $b$, then $\mathcal{H}_{\alpha}^{a}=\mathcal{H}_{\alpha}^{b}$ and $\mathcal{P}_{\alpha}^{a}=\mathcal{P}_{\alpha}^{b}$ for $\alpha=0, \infty$. This forces $\mathcal{H}_{1}^{a}=\mathcal{H}_{1}^{b}$ and $\mathcal{P}_{1}^{a}=\mathcal{P}_{1}^{b}$. 
$(1 \Rightarrow 4)$ We will prove a slightly more general result; namely, if $h_{a} \preceq h_{b}$, then there is a positive integer $j$ such that $r_{n}^{(a)} \geq r_{j n}^{(b)} / j$. There is no loss of generality in assuming $h_{x}\left(r_{n}^{(a)} / n\right)=h_{x}\left(r_{n}^{(b)} / n\right)=1 / n$. As $h_{a} \preceq h_{b}$ there is an integer $j>0$ such that

$$
h_{b}\left(\frac{r_{n}^{(a)}}{n}\right) \geq \frac{1}{j} h_{a}\left(\frac{r_{n}^{(a)}}{n}\right)=\frac{1}{j n}=h_{b}\left(\frac{r_{j n}^{(b)}}{j n}\right) .
$$

The increasingness of $h_{b}$ establishes the claim.

Corollary 4.5. If $h_{a} \equiv h_{b}$, then $C_{a}$ and $C_{b}$ have the same Hausdorff and packing dimensions.

Example 4.6. In [5, Ex. 4.6] a construction is given of a Cantor set $C_{a}$ which has Hausdorff and packing dimension $s$, but where $P_{0}^{s}\left(C_{a}\right)=\infty$. Thus if $C_{b}$ is any $s$-regular Cantor set, then $C_{a}$ and $C_{b}$ have the same dimensions, but not the same dimension partitions.

In Theorem 4.4 we proved that Cantor sets $C_{a}$ and $C_{b}$ have the same dimension partition if and only if the sequences $a$ and $b$ are weak tail-equivalent. We conclude the paper by determining when the class of sequences weak tail-equivalent to $a$ coincides with the class of sequences tail-equivalent to $a$.

Recall the example of the sequences $a=\left\{e^{-n}\right\}$ and $b=\left\{e^{-2 n}\right\}$ which are weak tail-equivalent, but not tail-equivalent. We can take $h_{a}(x)=|\log x|^{-1}$ and $h_{b}(x)=|\log \sqrt{x}|^{-1}$. These associated dimension functions are, of course, equivalent, but their inverse functions are neither equivalent nor doubling. As we see below, the latter is the key property.

Lemma 4.7. If $h \equiv g$ and $h^{-1}$ is doubling, then $h^{-1} \equiv g^{-1}$. In particular $g^{-1}$ is doubling.

Proof. Since $h \equiv g$ we have $c_{1} h\left(g^{-1}(y)\right) \leq y \leq c_{2} h\left(g^{-1}(y)\right)$ for suitable constants $c_{1}, c_{2}$. As $h^{-1}$ is doubling this implies that for some (probably different) positive constants, $c_{1} g^{-1}(y) \leq h^{-1}(y) \leq c_{2} g^{-1}(y)$, and therefore $g^{-1} \equiv h^{-1}$.

We can now prove the following theorem.

Theorem 4.8. Given a non-increasing, summable sequence a, let $W_{a}$ denote the equivalence class of sequences weak tail-equivalent to a. The following are equivalent:

(1) There exists $b \in W_{a}$ such that $h_{b}^{-1}$ is doubling.

(2) For every $b \in W_{a}, h_{b}^{-1}$ is doubling.

(3) Every $b \in W_{a}$ is tail-equivalent to $a$.

Proof. (1) $\Longleftrightarrow(2)$ follows from the previous lemma.

$(2) \Longrightarrow(3)$ Assume $b \in W_{a}$. Then $h_{a}^{-1} \equiv h_{b}^{-1}$ and both are doubling. These properties ensure that

$$
c_{1} h_{a}^{-1}\left(\frac{1}{n}\right) \leq h_{b}^{-1}\left(\frac{1}{n}\right) \leq c_{2} h_{a}^{-1}\left(\frac{1}{n}\right)
$$

and hence that there exist constants $c_{1}^{\prime}, c_{2}^{\prime}$ such that

$$
c_{1}^{\prime} \frac{r_{n}^{(a)}}{n} \leq \frac{r_{n}^{(b)}}{n} \leq c_{2}^{\prime} \frac{r_{n}^{(a)}}{n}
$$


that is, the sequence $a$ is tail-equivalent to $b$.

$(3) \Longrightarrow(1)$ We will prove this by contradiction. Suppose that $h_{a}^{-1}$ is not doubling. We will show there is a sequence $b \in W_{a}$ that is not tail-equivalent to $a$. Indeed, let $b=\left\{b_{k}\right\}$ be defined as

$$
b_{1}=a_{1}, \quad b_{2 k}=b_{2 k+1}=\frac{a_{k}}{2} .
$$

Then $r_{2 n}^{(b)}=r_{n}^{(a)}$, and by the definition of $h_{a}$, there exist constants $c_{1} \leq c_{2}$ such that

$$
\frac{r_{n}^{(b)}}{r_{n}^{(a)}}=\frac{1}{2} \frac{r_{n / 2}^{(a)}}{n / 2} \frac{n}{r_{n}^{(a)}} \geq \frac{1}{2} \frac{h_{a}^{-1}\left(2 c_{1} / n\right)}{h_{a}^{-1}\left(c_{2} / n\right)} \geq \frac{1}{2} \frac{h_{a}^{-1}\left(2 c_{1} / n\right)}{h_{a}^{-1}\left(c_{1} / n\right)},
$$

where in the last inequality we used the fact that $h_{a}^{-1}$ is increasing.

Since $h_{a}^{-1}$ is both increasing and non-doubling, it follows that

$$
\sup _{n} \frac{h_{a}^{-1}(2 / n)}{h_{a}^{-1}(1 / n)}=\infty \text {. }
$$

Using this in equation (4.1) we conclude that the ratios $r_{n}^{(b)} / r_{n}^{(a)}$ are not bounded above and thus the sequences $a$ and $b$ are not tail-equivalent. However, as they are clearly weak tail-equivalent, $b \in W_{a}$. This contradicts (3).

\section{REFERENCES}

[1] A.S. Besicovitch and S.J. Taylor, On the complementary intervals of a linear closed set of zero Lebesgue measure, J. London Math. Soc. 29(1954), 449-459. MR0064849 (16:344d)

[2] C. Cabrelli, F. Mendivil, U. Molter and R. Shonkwiler, On the h-Hausdorff measure of Cantor sets, Pac. J. of Math. 217(2004), 29-43. MR2105765 (2005h:28013)

[3] C. Cabrelli, U. Molter, V. Paulauskas and R. Shonkwiler, The Hausdorff dimension of $p$ Cantor sets, Real Anal. Exchange 30(2004/05), no. 2, 413-433. MR2177411 (2006g:28012)

[4] K. Falconer, Techniques in fractal geometry, Wiley and Sons, Chichester, 1997. MR1449135 (99f:28013)

[5] I. Garcia, U. Molter and R. Scotto, Dimension functions of Cantor sets, Proc. Amer. Math. Soc. 135(2007), 3151-3161. MR2322745 (2008i:28004)

[6] C. A. Rogers, Hausdorff measures, Cambridge Math Library, Cambridge University Press, Cambridge, 1998. MR1692618(2000b:28009)

[7] C. Tricot, Two definitions of fractional dimension, Math. Proc. Camb. Phil. Soc. 91(1982), 57-74. MR633256 (84d:28013)

[8] S. J. Taylor and C. Tricot, Packing measure and its evaluation for a Brownian path, Trans. Amer. Math. Soc. 288(1985), 679-699. MR776398 (87a:28002)

Departamento de Matemática, Facultad de Ciencias Exactas y Naturales, Universidad de Buenos Aires, Pabellón I, Ciudad Universitaria, C1428EGA C.A.B.A., Argentina - AND - CONICET, ARgEnTINA

E-mail address: cabrelli@dm.uba.ar

Department of Pure Mathematics, University of Waterloo, Waterloo, on, Canada

E-mail address: kehare@uwaterloo.edu

Departamento de Matemática, Facultad de Ciencias Exactas y Naturales, Universidad de Buenos Aires, Pabellón I, Ciudad Universitaria, C1428EGA C.A.B.A., Argentina - AND - CONICET, ARgentina

E-mail address: umolter@dm.uba.ar 